\title{
Drift Velocities and Momentum Distributions of Hot Carriers in MOSFETs at Low Supply Voltages
}

\author{
C. C. C. Leung and P. A. Childs
}

School of Electronic and Electrical Engineering, The University of Birmingham
P.O. Box 363, Birmingham, B15 2TT, UNITED KINGDOM

\begin{abstract}
In this paper we determine the momentum distribution and drift velocity of hot carriers in silicon under the condition of finite supply energy. We show that the momentum distribution varies between the nearly isotropic in high field to strongly anisotropic when the carrier's energy is comparable to the supply energy.
\end{abstract}

\section{Introduction}

Hot Carriers have long been known to create serious reliability problems for short channel MOSFETs. It was believed that scaling of the supply voltage would virtually eliminate the problem. However, hot carrier currents and degradation have been observed at drain voltages of 3.3V and below [1]. Recent Monte Carlo simulations of hot electron transport in MOSFETs operating at low drain voltages have indicated that the high energy tail of the electron distribution, the most important quantity in determining reliability, is determined by the supply voltage rather than local electric fields [2]. In this paper we show how the momentum distribution and drift velocities of hot carriers in the high energy tail of the distribution relate to hot carrier phenomena.

The momentum distribution of hot carriers is obtained by solving the Boltzmann Transport equation using a modification of the Chambers' path integral solution [3]. This form of the Boltzmann transport equation can be solved efficiently by an iterative method developed by the authors $[4,5]$. At low drain voltage a one dimensional solution can be obtained in only seconds of CPU time on a SUN sparcstation. 


\section{Momentum Distributions and Drift Velocities}

Earlier studies of electron distributions by Baraff [6] and Keldysh [7] provide accurate solutions to the Boltzmann transport equation in the case of uniform fields in infinitely long samples. However, these analyses are not applicable when the supply energy is finite. In this case the distributions vary between the nearly isotropic in high field and low energy regions to strongly anisotropic close to the supply energy. The high energy drift velocities are important when the electric field is in the direction of the potential barrier, for example in substrate hot electron injection, where they significantly modify the injection current. To illustrate the method the BTE is solved over a potential profile obtained from MINIMOS. The distribution functions obtained from the analytical solution of the BTE, derived by Keldysh for uniform fields [7], are used as boundary conditions in the low field region.

\section{Results and Discussion}

Figure 1 shows the hot carrier distribution obtained at the edge of the drain depletion region. The momentum distribution of hot electrons obtained at two points, one at the average energy and a second in the tail, is shown as a polar plot in figure 2. The magnitude of the vector represents the probability of finding an electron travelling at the angle made by the vector with respect to the electric field. At low energies the momentum distribution is nearly spherical symmetric, whilst in the tail it is strongly anisotropic. The effect of the momentum distribution on drift velocity is shown in figure 3. Around the average energy the velocity of the electrons in the field direction increases as energy $y^{1 / 2}$ and the average drift velocity is saturated. At high energies the velocity in the field direction increases sharply as the distribution becomes increasingly anisotropic. Electrons in this region gain energy by making few collisions with phonons, effectively travelling by 'very lucky drift'. It is, therefore, apparent that although the probability of finding hot carriers falls off rapidly due to the finite supply energy, the drift velocity of these carriers will significantly increase the current density as measured in the direction of the electric field.

It is interesting to note the electron distribution developed at an energy above that of the supply. In this region the energy distribution is identical to that formed by the boundaries. However, the momentum distribution is modified by the field and remains highly anisotropic. 


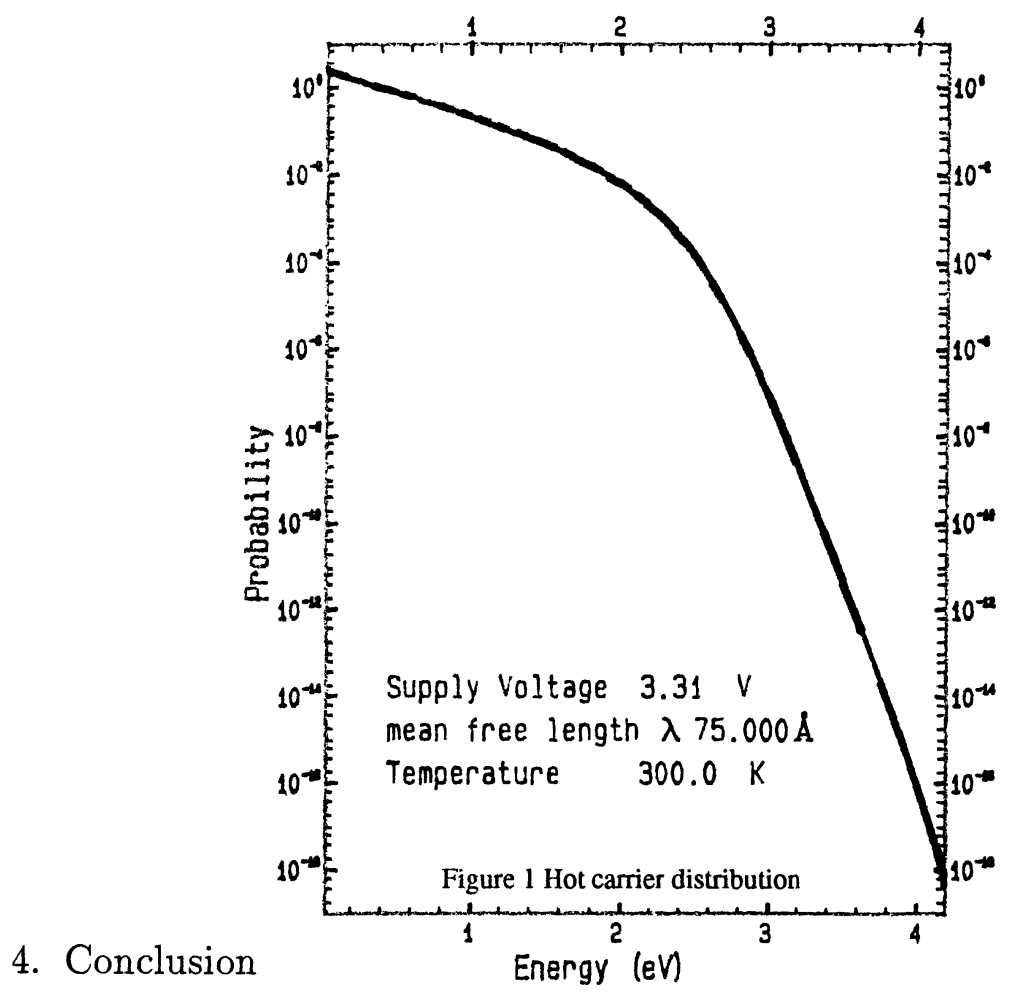

In summary the paper shows the importance of hot carrier momentum distributions and drift velocities on hot carrier currents at low supply voltages. In obtaining the hot carrier distributions the application of a new and highly efficient technique for solving the spatially dependent BTE has been demonstrated.

Acknowledgment: This work is part of the IED1939 project funded by the DTI and SERC

\section{References}

1. J E Chung, M-C Jeng, J E Moon, P-K Ko and C Hu, 'Low-Voltage Hot-Electron Currents and degradation in Deep-Submicron MOSFETs', IEEE Trans. Elec. Dev. 37 No. 7, p.1651 (1990)

2. F Venturi, E Sangiorgi, and B Ricco, 'The Impact of Voltage Scaling on Electron Heating and Device Performance of Submicrometer MOSFETs', IEEE Trans Elec. Dev., 38 No. 8, p. 1895 (1991)

3. R. G. Chambers, Proc. Phys. Soc. A65, 458 (1952)

4. C C C Leung and P A Childs, 'Hot Carrier Distributions in Silicon Determined by Path Integral Solution of the Boltzmann Transport Equation', Proc. IEDMS, Taiwan p.209 (1992)

5. C C C Leung and P A Childs, 'Spatially Transient Hot Electron Distributions in Silicon Determined from the Chambers Path Integral Solution of the Boltzmann Transport Equation', Solid State Electron, 36, No. 7, 1001 (1993) 
6. G. A. Baraff, 'Distribution Functions and Ionization Rates for Hot Electrons in Semiconductors', Phys. Rev. 133, A26 (1964)

7. L. V. Keldysh, 'Concerning the theory of Impact Ionisation in Semiconductors', Soviet Physics JETP, 21, 1135 (1965)
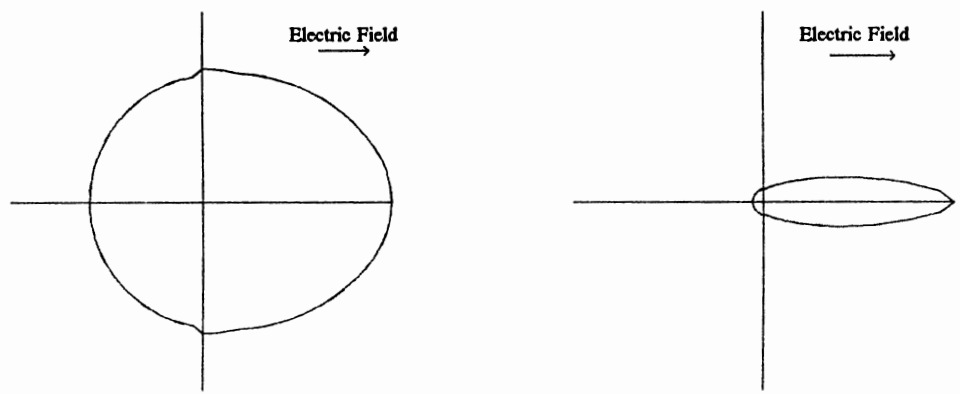

Figure 2 Momentum distribution of hot electrons at an average energy(left) and in the tail (right) of

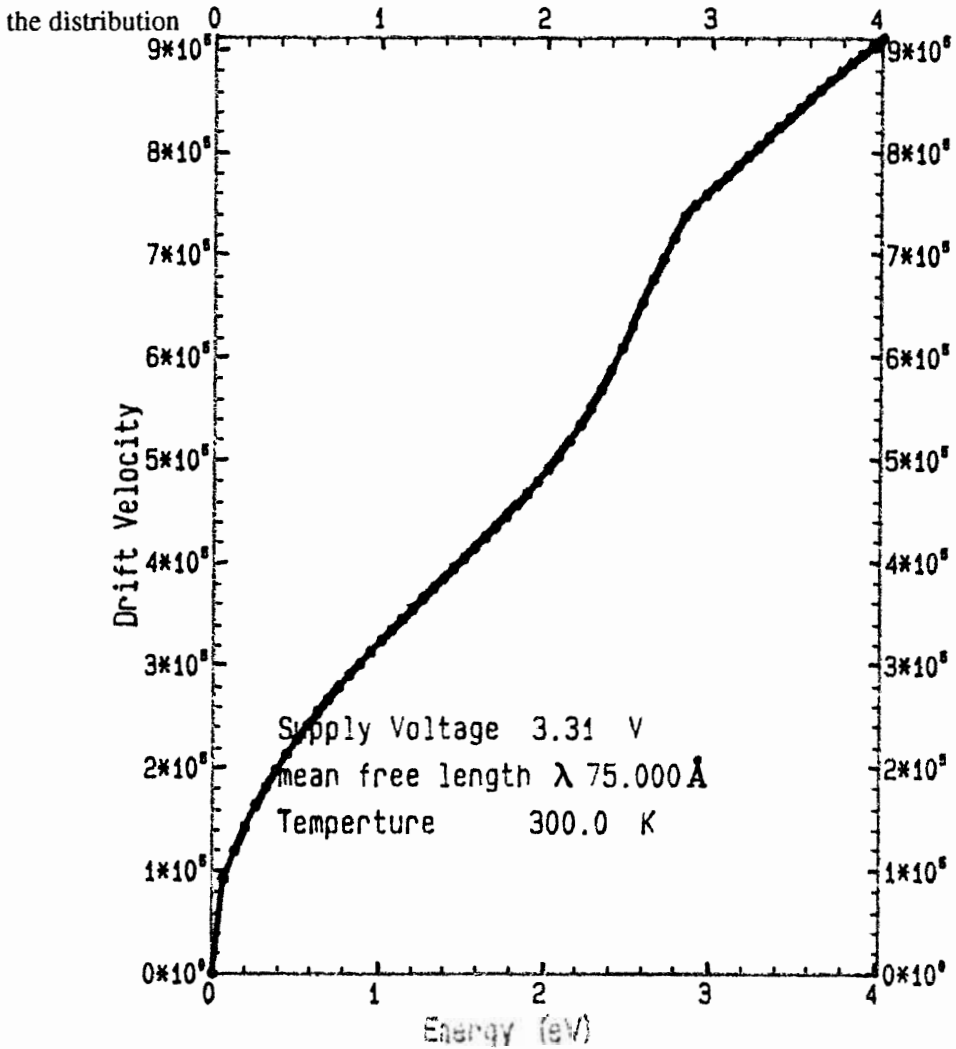

Figure 3. Drift velocity as a function of clectron's energy 